\title{
Overloads Management in Active Radial Distribution Systems: an Optimization Approach Including Network Switching
}

\author{
Ilya Bilibin, Florin Capitanescu, Juergen Sachau \\ University of Luxembourg, Faculty of Science, Technology and Communication, \\ Interdisciplinary Centre for Security, Reliability and Trust (SnT) \\ ilya.bilibin@uni.lu, florin.capitanescu@uni.lu,juergen.sachau@uni.lu
}

\begin{abstract}
This paper focuses on the overloads management in active radial distribution systems that host a significant amount of distributed generation (DG). In order to possibly reduce the amount of generation curtailed to remove congestion, and hence harvest as much renewable energy as possible, we propose a centralized optimization approach that includes the option to use remotely controlled grid switches and breakers so as to transfer distributed generation between feeders. As switching actions are modeled as binary variables, to mitigate the computational burden posed by the original mixed integer nonlinear programming (MINLP) problem we build upon a very convenient existing optimization framework that, for a radial distribution grid, transforms precisely the problem into a much simpler mixed integer quadratically constrained (MIQC) program. Although the approach focuses on overload removal in course of optimization it also inhibits violation of other operational constraints such as voltage limits. We prove the interest and feasibility of our approach using a snapshot of a 33bus benchmark distribution system and of a 61-bus real-life distribution system model, whereas future work is planned to assess the benefits of the method using time-series.
\end{abstract}

Index Terms - active distribution system, congestion management, distributed generation, optimal power flow, network switching, smart grid.

\section{INTRODUCTION}

Driven mainly by more and more stringent environmental constraints, nowadays, many distribution systems (DSs) host increasing amounts of distributed generation (DG) $[1,2]$. Beyond some limit of penetration the DG units can pose significant operational problems to traditionally passive DSs (e.g. overloads, over-voltages, protection malfunction, etc.). There are two philosophies for determining the upper limit on the amount of DG penetration [1]:

- conservative passive DSs based on the "fit-and-forget" principle i.e. a new DG is accommodated only if this does not lead to operational constraints violation under worst operating scenario, which corresponds generally to minimum load and maximum feed-in. This approach is very simple and has a low cost but may prevent achieving the required green energy target or limits harvesting other benefits of DG;

- active DSs (which represents an intermediate step towards smart grids), where in order to significantly increase the amount of DG penetration, some centralized [3] or distributed [4] control schemes are put in place, together with additional communication, measuring, and metering devices, to manage DG outputs possibly together to other control means (e.g. substation transformer voltage setpoint, shunt reactive power, load deferral, remote network reconfiguration, etc.). Such schemes are very promising, one practical implementation being already reported in [5].

In this work we focus on the active $D S$ paradigm in which we assume a centralized management of network congestions [3]. This relies in turn on the output of a state estimator, which does not require a substantial number of additional measurements being deployed [6] and may further take advantage from the data stemming from home smart meters, which installation is underway in several countries. In this paradigm there is a need to devise fast tools tailored to DSs providing optimal control actions to remove overload and voltage congestions in real-time. We assume in this paper that overload and voltage congestions are managed separately and successively. We further focus on the overloads congestion and assume that voltage congestions can be addressed by the classical Volt/VAR control.

Optimal power flow (OPF) [7] is an important tool to manage congestions in both transmission $[7,8]$ and distribution systems [3]. In the DSs it provides optimal generation curtailment to remove congestions according to a given objective function (e.g. minimum MW curtailed) or operational rules (e.g. last-in, first-off) [3]. This OPF is generally formulated as a nonlinear programming problem. However, in modern distribution systems there coexist manually controlled switches together with remotely controlled switches and breakers. Furthermore, in their transition towards active DSs, it is expected that distribution systems will be equipped at strategic locations with an 
increasing number of remotely controlled switches and/or breakers (e.g. for maintaining protection coordination in the presence of DG) that will greatly facilitate the fast grid control and improve reliability.

The main contribution of this work is to extend a conventional OPF for distribution system [3] so as to include network switching as control variable, which may lead to a smaller curtailment of DG units as they can be transferred between feeders in case of congestion. However, this leads to posing a mixed integer nonlinear programming (MINLP) problem, whereas solvers of this class of optimization problems are not able to comply with the stringent time requirements of the real-time control $[9,10]$. Fortunately, as most distribution systems are operated radially, there is possible to reformulate the MINLP problem as a more tractable equivalent mixed integer quadratically constrained (MIQC) problem, as demonstrated in [11] for the power losses minimization by means of network reconfiguration. In this work we further adapt the model in [11] to the problem of overloads management. In particular we model properly the case where DG units responsible of overload are requested to completely shut down.

\section{OPTIMIZATION MODEL FOR OVERLOAD MANAGEMENT}

Our optimization problem adopts the model proposed in [11] for radial distribution networks, which relies in turn on the power flow model for radial distribution systems proposed in [12]. This model consists, for a grid with $n+1$ buses (we assume here for simplicity a single substation but the model is generic to any number of substations), in replacing the $2 n$ conventional nonlinear power flow equations with a set of $3 n$ equations ( $2 n$ linear and $n$ quadratic). This can be done by replacing the conventional complex voltage unknowns $\left(V_{i}, \theta_{i}\right)$ with two variables per branch $\left(W_{i j}\right.$ and $\left.T_{i j}\right)$ and one per bus $\left(U_{i}\right)$ as follows:

$$
\begin{gathered}
U_{i}=V_{i}^{2}, \\
W_{i j}=V_{i} V_{j} \cos \left(\theta_{i}-\theta_{j}\right), \\
T_{i j}=V_{i} V_{j} \sin \left(\theta_{i}-\theta_{j}\right) .
\end{gathered}
$$

The new variables are related among each other as

$$
U_{i} U_{j}=W_{i j}^{2}+T_{i j}^{2} \text {. }
$$

At the solution of these power flow equations traditional voltage unknowns can be straightforwardly retrieved: $V_{i}$ from (1), and $\theta_{i}$ from (2) or (3) by exploring the grid tree downward from the slack bus substation.

These new variables enable active and reactive power flows and the square branch currents to be expressed linearly (a $\pi$-representation of branches is used as shown in Fig. 1):

$$
\begin{gathered}
P_{i j}=g_{i j} U_{i}-g_{i j} W_{i j}+b_{i j} T_{i j}, \\
Q_{i j}=\left(b_{i j}-b_{i j}^{s h}\right) U_{i}-b_{i j} W_{i j}-g_{i j} T_{i j}, \\
I_{i j}^{2}=\left(g_{i j}^{2}+b_{i j}^{2}\right)\left(U_{i}+U_{j}-2 W_{i j}\right)+
\end{gathered}
$$

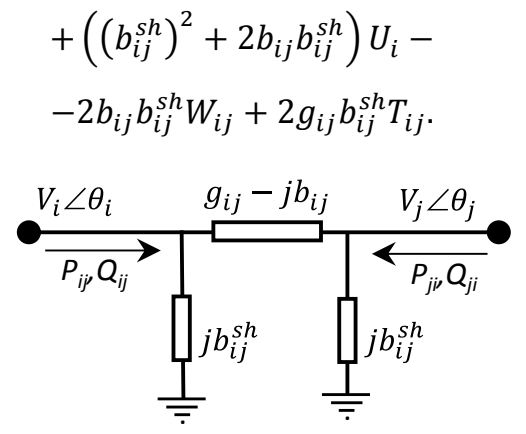

Fig. 1. Branch model.

The goal of the overload management procedure is to minimize the active power curtailment of the DG units:

$$
\min \sum_{g}\left(P_{g}^{0}-P_{g}\right)
$$

where $P_{g}^{0}$ is the power produced by the DG $g$ at a given time.

The decision variables are: status on/off and the active/reactive powers of DG units, substation generator active/reactive power, and the status of remotely controlled sectionalizing switches, tie switches, and breakers.

The constraints of the optimization problem are as follows. The active and reactive power flows equations in every bus $i$ are given by:

$$
\begin{aligned}
& P_{g}^{\mathrm{i}}-P_{c}^{\mathrm{i}}=\sum_{j} P_{i j}, \\
& Q_{g}^{\mathrm{i}}-Q_{c}^{\mathrm{i}}=\sum_{j} Q_{i j},
\end{aligned}
$$

where $P_{i j}$ and $Q_{i j}$ are given by the expressions (5) and (6). Active/reactive power flows and square currents are limited at both sides of every branch $i j$ :

$$
\begin{gathered}
-S_{i j} P_{i j}^{\max } \leq P_{i j} \leq S_{i j} P_{i j}^{\max }, \\
-S_{i j} Q_{i j}^{\max } \leq Q_{i j} \leq S_{i j} Q_{i j}^{\max }, \\
I_{i j}^{2} \leq K_{i}\left(1-S_{i j}\right)+S_{i j}\left(I_{i j}^{\max }\right)^{2},
\end{gathered}
$$

where $S_{i j}$ models the status of the line switch $i j\left(S_{i j}=1\right.$ if the line is connected and $S_{i j}=0$ if the line is disconnected).

When the status of the line $S_{i j}$ is taken into account the expression (4) transforms into:

$$
\begin{gathered}
-K_{e q}\left(1-S_{i j}\right) \leq U_{i} U_{j}-W_{i j}^{2}-T_{i j}^{2} \leq \\
\leq K_{e q}\left(1-S_{i j}\right), \\
-K_{W}\left(1-S_{i j}\right) \leq W_{i j}-W_{j i} \leq K_{W}\left(1-S_{i j}\right), \\
-K_{T}\left(1-S_{i j}\right) \leq T_{i j}-T_{j i} \leq K_{T}\left(1-S_{i j}\right),
\end{gathered}
$$

the constants appearing in constraints (13)-(16) being properly chosen as explained in [11]. 
The active (respectively reactive) power of DG $g$ is limited by its minimum and current (respectively maximum) power of the generator as:

$$
\begin{gathered}
P_{g}^{\min } S_{g} \leq P_{g} \leq P_{g}^{0} S_{g}, \\
Q_{g}^{\min } S_{g} \leq Q_{g} \leq Q_{g}^{\max } S_{g},
\end{gathered}
$$

where $S_{g}$ is the status of the generator $g\left(S_{g}=1\right.$ if the generator is connected and $S_{g}=0$ if it is shut down).

Note that in this approach we consider that DG can shift their reactive power so as to reduce the overall amount of active power curtailed but other typical reactive power control modes of DG (e.g. constant power factor and in particular unitary power factor) can be taken into account straightforwardly.

For the equivalent generators at substations the following box constraints are considered:

$$
\begin{gathered}
P_{g}^{\min } \leq P_{g} \leq P_{g}^{\max }, \\
Q_{g}^{\min } \leq Q_{g} \leq Q_{g}^{\max } .
\end{gathered}
$$

Voltage magnitude is limited in every bus $i$ :

$$
\left(V_{i}^{\min }\right)^{2} \leq U_{i} \leq\left(V_{i}^{\max }\right)^{2} .
$$

In case the generator $g$ is able of controlling voltage level in the bus $i$ an additional constraint is considered:

$$
U_{i}=\left(V_{g}^{\text {nom }}\right)^{2} .
$$

Furthermore, to ensure the radiality of the distribution network the following necessary constraint is added, where $S_{i j}^{0}$ is the initial status connected/disconnected of the line $i j$ :

$$
\sum_{i j} S_{i j}=\sum_{i j} S_{i j}^{0}
$$

This constraint may be however insufficient to ensure radiality in the presence of zero-injection nodes [11]. To avoid adding new variables and constraints to ensure radiality as in [11], rendering thereby the model more complex, we adopt a practical solution and replace each zero-injection bus with a very small reactive power load (of value slightly above the power flow convergence tolerance), change which practically does not affect the final result of the optimization.

Notice that the congestion management optimization model (5), (6), (8)-(23) constitutes a very convenient MIQC problem, the only nonlinear (but quadratic) constraints are (14). The differences compared to the model in [11] are: the objective function, control variables related to DG, and constraints (17)(20), (23).

\section{SIMULATION RESULTS}

\section{A. Simulation assumptions}

The MIQCP optimization model has been developed in GAMS version 23.9.3 [13] and uses the simple branch and bound (SBB) solver. All tests have been performed on a PC of 2.8-GHz and 4-Gb RAM.
For the sake of assessing the efficiency of the optimization engines we assume that for all test systems all switches are remotely controlled.

We consider three simulation cases differing in the type of control variables and values of thermal limits as follows:

- case A where one removes the congestion only by DG curtailment;

- case B where one allows switching actions as additional control means;

- case $\mathrm{C}$ where, compared to case $\mathrm{B}$, for further assessment of the optimization engines performances, one limits drastically the thermal ratings of tie lines.

We consider voltage limits of $0.95 / 1.05$ p.u. at all nodes.

\section{B. Tests on the 33-bus distribution network}

We first illustrate the approach on a $12.66 \mathrm{kV}$ benchmark distribution grid [14] with the following characteristics: 33 buses, one substation, 37 lines, 32 sectionalizing switches, and 5 tie switches. Figure 2 shows the one-line diagram of this network. The overall load in the base case is $3.715 \mathrm{MW}$ and 2.3 MVar.

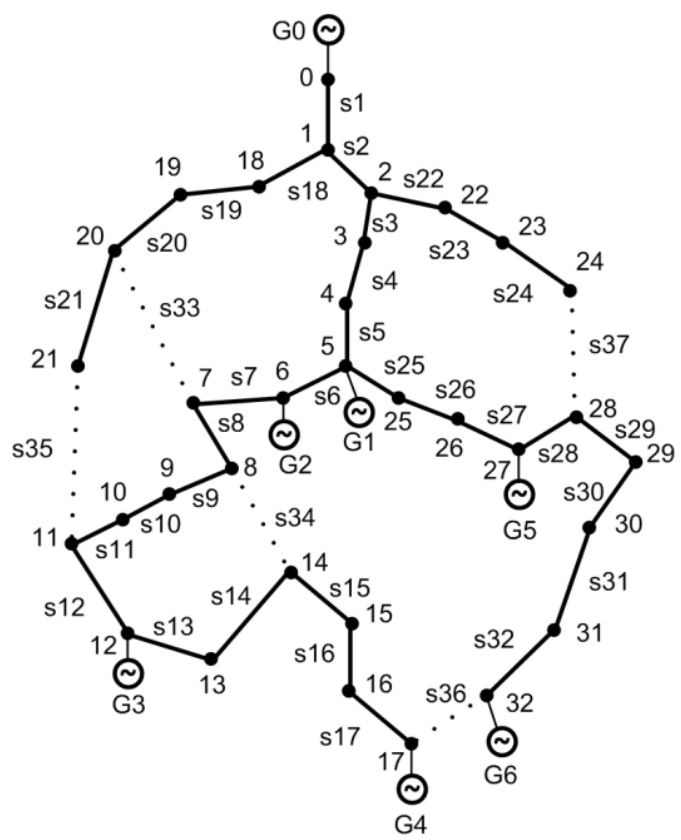

Fig. 2. One-line diagram of the 33-bus system with DG.

TABLE I. GENERATORS ACTIVE/REACTIVE POWERS (MW/MVAR)

\begin{tabular}{|c|c|c|c|c|c|c|}
\hline Gen & $\mathrm{P}_{0}$ & $\mathrm{Q}_{0}$ & $\mathrm{P}_{\min }$ & $\mathrm{P}_{\max }$ & $\mathrm{Q}_{\min }$ & $\mathrm{Q}_{\max }$ \\
\hline \hline G0 & 0.188 & 1.754 & 0.0 & 9.0 & -1.0 & 8.0 \\
\hline G1 & 0.6 & 0.1 & 0.1 & 0.6 & -0.2 & 0.1 \\
\hline G2 & 0.6 & 0.1 & 0.1 & 0.6 & -0.2 & 0.1 \\
\hline G3 & 0.6 & 0.1 & 0.1 & 0.6 & -0.2 & 0.1 \\
\hline G4 & 0.6 & 0.1 & 0.1 & 0.6 & -0.2 & 0.1 \\
\hline G5 & 0.6 & 0.1 & 0.1 & 0.6 & -0.2 & 0.1 \\
\hline G6 & 0.6 & 0.1 & 0.1 & 0.6 & -0.2 & 0.1 \\
\hline
\end{tabular}


In order to test our approach we consider 6 identical DG units (G1 to G6) connected to this grid together with the equivalent generator G0 modeling the transmisison system. Table I provides the initial active/reactive power of these DG units as well as their physical power limits.

We set the thermal limit of all to 2 MVA, except of line 01 which has a limit of 10 MVA and 4-5 which has a limit of 1 MVA. Given these limits the line 4-5 gets overloaded due to the significant amount of feed-in, its limit being exceeded with 0.76 MVA.

Table II presents numerical results with the approach in three cases. In case A the optimal DG curtailment, which creates a counter-flow in line 4-5 and removes congestion, consists of reducing the output of G1 to its minimum value and slightly reducing the output of $\mathrm{G} 2$, those changes being balanced by the equivalent generator G0. In case B one can observe that by opening the sectionalizing switch s10 and closing the tie switch s35 the power from two DG units (G3 and G4) is rerouted and congestion disappears without curtailing any generator, which proves the advantage of our approach. In case $\mathrm{C}$ the thermal limits of the 5 tie-lines have been decreased artificially to 0.3 MVA. Due to this strong limitation the congestion cannot be removed only by switching actions, and some generation is still curtailed, however to a less extent than in the case without switching. We conclude that thanks to the additional degrees of freedom provided by remotely controlled network switches the DG is curtailed less which demonstrates the benefit of the approach.

TABLE II. GENERATORS ACTIVE POWER ADJUSTMENT (MW) AND SWITCHING ACTIONS TO REMOVE CONGESTION

\begin{tabular}{|c|c|c|c|}
\hline Controls & Case A & Case B & Case C \\
\hline \hline G1 & -0.5 & & \\
\hline G2 & -0.014 & & -0.237 \\
\hline G3 & & & \\
\hline G4 & & & \\
\hline G5 & & & \\
\hline G6 & & & s8, $\underline{\mathrm{s} 35}$ \\
\hline switches & - & s10, $\underline{\mathrm{s} 35}$ & 0.237 \\
\hline \hline $\begin{array}{c}\text { Objective } \\
\text { (MW) }\end{array}$ & 0.514 & 0.0 & \\
\hline Time (s) & 0.21 & 1.35 & 23.03 \\
\hline
\end{tabular}

\section{Results on the 61-bus CREOS distribution network}

The network model was provided by the Luxembourgish grid operator (CREOS) and represents a 61-bus simplified subsystem of $20 \mathrm{kV}$ system fed by one substation. The system contains 2 feeders, 63 lines, 60 sectionalizing switches, and 3 tie switches. The total load is of 11.69 MW and 2.37 MVAr. We consider a virtual operating scenario where 20 distributed generators of various sizes are connected to the grid.

We assume that due to the significant power feed-in one line in the first main feeder gets congested.
Table III presents the control actions to remove congestion used in three cases. In case A the optimal DG curtailment consists of shutting-down G1, and reducing the output of G2 and G3. In case B, the congestion is removed by simply operating on switches: one can observe that by opening the sectionalizing switch s10 and closing the tie switch s35 the power from two DG units (G3 and G4) is rerouted and congestion disappears without curtailing any DG, which proves again the benefit of our approach. In case $\mathrm{C}$ the thermal limits of the 3 tie-lines have been decreased to 1.0 MVA. Due to this strong limitation the congestion cannot be removed only by switching actions, and some generation is still curtailed, however to a less extent than in the case without switching.

TABLE III. GENERATORS ACTIVE POWER ADJUSTMENT (MW) AND SWITCHING ACTIONS TO REMOVE CONGESTION

\begin{tabular}{|c|c|c|c|}
\hline Controls & Case A & Case B & Case C \\
\hline \hline G1 & $-1.18^{*}$ & & -0.86 \\
\hline G2 & -0.94 & & \\
\hline G3 & -0.58 & & \\
\hline G4 & & & -0.31 \\
\hline G5 & & & -0.53 \\
\hline G6 & & & \\
\hline$\ldots$ & & & \\
\hline G20 & & & $s 17, \underline{s 63}$ \\
\hline switches & - & s1, $\underline{622}$ & 1.72 \\
\hline \hline $\begin{array}{c}\text { Objective } \\
\text { (MW) }\end{array}$ & 2.7 & 0.0 & \\
\hline Time (s) & 0.43 & 17.8 & 48.9 \\
\hline
\end{tabular}

We further decrease the thermal limit of the congested circuit from 3 MVA to 2 MVA and report the results of the three cases in Table IV. As expected, in cases A and $\mathrm{C}$ the optimal amount of DG curtailment increases with about 1 MW. We noticed that in case A three generators are shut down (G1, G2, and G3).

TABLE IV. GENERATORS ACTIVE POWER ADJUSTMENT (MW) AND SWITCHING ACTIONS TO REMOVE CONGESTION IN A MORE SEVERE CASE

\begin{tabular}{|c|c|c|c|}
\hline Controls & Case A & Case B & Case C \\
\hline \hline G1 & $-1.18^{*}$ & & $-1.18^{*}$ \\
\hline G2 & $-1.04^{*}$ & & -0.58 \\
\hline G3 & $-1.00^{*}$ & & \\
\hline G4 & -0.15 & & -0.64 \\
\hline G5 & -0.33 & & -0.31 \\
\hline$\ldots$ & & & \\
\hline G20 & & & \\
\hline switches & - & s9, s62 & s17, s63 \\
\hline \hline $\begin{array}{c}\text { Objective } \\
\text { (MW) }\end{array}$ & 3.70 & 0.0 & 2.71 \\
\hline Time (s) & 0.49 & 10.7 & 49.3 \\
\hline
\end{tabular}




\section{Computational effort}

Looking closely to the computational times reported in Tables II, III, and IV one can remark that as long as both types of control actions (i.e. switches and DG output) are not interacting together (as in cases A and B) the optimal solution is obtained acceptably fast. On the other hand, as expected, the case $\mathrm{C}$ takes a bit longer time due to the strong interaction between both types of controls and very tight constraints. However, the program can be stopped after a desired elapsed time and still provide a better solution than that obtained only by DG output curtailment. Furthermore, our assumption that all switched are remontely controlled leads to a significantly large combinatorial space. The computational time is expected to highly decrease (e.g. being just few order of magnitude larger than in case A) as only a limited number of switches are considered remotely controlled, which is a reasonable assumption in most distribution companies due to the trade-off between investments and reliability improvement.

\section{CONCLUSIONS AND FUTURE WORKS}

This paper has proposed a centralized optimization approach for the thermal congestion management in active radial distribution systems. The main feature of the scheme is that it allows the joint optimization of DG output and remotely controlled grid switches and breakers, which can lead in some cases to reduce the amount of curtailed generation, thanks to the transfer of distributed generators between feeders. We have proven the feasibility and efficiency of the approach for a snapshot of two test systems, whereas the application of the method on-line in a closed-loop fashion or in a (pseudo) realtime environment by means of time-series analysis [3],[4],[5] requires further investigation.

The obtained computational times are reasonable for the application of the approach in a real-time environment. Although the problem is formulated in a very convenient way as a MIQCP we noticed that the solvers in GAMS tailored for this type of optimization problem (e.g. CPLEX, MOSEK, GUROBI, XPRESS, etc.) cannot be used as they require the matrix of quadratic constraints to be positive semi-definite, requirement which is not met by this optimization model. Nonetheless, thanks to the linear/quadratic problem formulation, the MINLP solver SBB, which was also employed in [11], needs to solve simpler quadratically constrained sub-problems instead of the nonlinear subproblems in the original MINLP problem formulation.

Future work will assess how the approach scales with the problem size especially in the context of larger combinatorial spaces. Furthermore, we will conduct future research towards the development of an approach coordinating the management of both thermal and voltage congestions. Future work is also planned to extend the optimization model by imposing further constraints on fault current levels so as to preserve the feeders' protection selectivity when transferring DG units from one feeder to another.

\section{ACKNOWLEDGMENTS}

This work is conducted as part of the "Reliable and Efficient Distributed Electricity Generation in Smart Grids" (REDESG) project within the "CREOS-SnT Framework Program". The authors are supported by the Fonds National de la Recherche, Luxembourg (C11/SR/1278568).

\section{REFERENCES}

[1] A. Keane, L.F. Ochoa, C.LT. Borges, G.W. Ault, A. D. AlarconRodriguez, R. A. F. Currie, F. Pilo, C. Dent, and G.P. Harrison, "State-ofthe-Art Techniques and Challenges Ahead for Distributed Generation Planning and Optimization", IEEE Transactions on Power Systems, in press.

[2] P.S. Georgilakis and N.D. Hatziargyriou, "Optimal Distributed Generation Placement in Power Distribution Networks: Models, Methods, and Future Research", IEEE Transactions on Power Systems, in press.

[3] M.J. Dolan, E.M. Davidson, I. Kockar, G.W. Ault, and S.D.J. McArthur, "Distribution power flow management utilizing an online optimal power flow technique", IEEE Transactions on Power Systems, Vol. 27, No. 2, pp. 790-799, May 2012.

[4] T. Sansawatt, L.F. Ochoa, and G.P. Harrison, "Smart decentralized control of DG for voltage and thermal constraint management", IEEE Transactions on Power Systems, Vol. 27, No. 3, pp. 1637-1645, August 2012.

[5] R.A.F. Currie, C.E.T. Foote, G.W. Ault, and J.R. McDonald, "Active power flow management utilising operating margins for the increased connection of distributed generation", IET Generation, Transmission, Distribution, vol. 1, No. 1, pp. 197-202, January 2007.

[6] R. Singh, B.C. Pal, R.A. Jabr, and R.B. Vinter, "Meter placement for distribution system state estimation: an ordinal optimization approach", IEEE Transactions on Power Systems, vol. 26, no. 4, pp. 2328-2335, 2011.

[7] F. Capitanescu, J.L. Martinez Ramos, P. Panciatici, D. Kirschen, A. Marano Marcolini, L. Platbrood, and L. Wehenkel, "State-of-the-art, challenges, and future trends in security-constrained optimal power flow", Electric Power System Research, vol. 81, no. 8, pp 1731-1741, 2011.

[8] R.D. Christie, B.F. Wollenberg, and I. Wangensteen, "Transmission management in the deregulated environment", Proc. of the IEEE, Vol. 88, No. 2, pp. 170-195, 2000

[9] M. Lavorato, J.F. Franco, M.J. Rider, and R.Romero, “Imposing Radiality Constraints in Distribution System Optimization Problems", IEEE Trans. on Power Systems, vol. 27, no. 1, pp. 172-180, 2012.

[10] L. Platbrood, S. Fliscounakis, F. Capitanescu, P. Panciatici, C. Merckx, and M. Ortega-Vazquez, "Deliverable D3.2: development of prototype software for system steady-state optimization of the European transmission system", PEGASE project, available on-line at http://www.fp7-pegase.com, 2011.

[11] E. Romero Ramos, J. Riquelme Santos, and J. Reyes, “A simpler and exact model for the computation of the minimal power losses tree", Electric Power Systems Research, Vol. 80, No. 5, pp. 562-571, May 2010.

[12] A. Gomez Exposito and E. Romero Ramos, "Reliable load flow technique for radial distribution networks", IEEE Trans. on Power Systems, vol. 14, no. 3, August 1999, pp. 1063-1069.

[13] B.A. McCarl, "GAMS User Guide", Version 23.8, 2012. Available online: www.gams.com.

[14] M.E. Baran and F.F. Wu, "Network Reconfiguration in Distribution Systems for Loss Reduction and Load Balancing", IEEE Trans. Power Delivery, Vol. 4, No. 2, 1989, pp. 1401-1497. 